\title{
CLASSIFICAÇÃO DE RESÍDUOS DE MADEIRA TRATADA COM PRESERVATIVOS À BASE DE ARSENIATO DE COBRE CROMATADO E DE BORO/FLÚOR
}

\author{
Suzana Frighetto Ferrarini*, Heldiane Souza dos Santos, Luciana Gampert Miranda, Carla M. N. Azevedo e Marçal J. \\ R. Pires \\ Faculdade de Química, Pontifícia Universidade Católica do Rio Grande do Sul, Av. Ipiranga, 6681, 90619-900 Porto Alegre - RS, \\ Brasil \\ Sandra Maria Maia \\ Instituto de Química, Universidade Federal do Rio Grande do Sul, Av. Bento Gonçalves, 9500, 91501-970 Porto Alegre - RS, Brasil
}

Recebido em 4/1/12; aceito em 6/6/12; publicado na web em 24/8/12

\begin{abstract}
CLASSIFICATION OF WASTE WOOD TREATED WITH CHROMATED COPPER ARSENATE AND BORON/FLUORINE PRESERVATIVES. Classification of waste wood treated with chromated copper arsenate (CCA) and boron/fluorine preservatives, according to NBR 10004, was investigated. The leaching test (ABNT NBR 10005) for As and Cr, and solubilization test (ABNT NBR 10006) for F, were applied to out-of-service wooden poles. Concentrations of As and $\mathrm{Cr}$ in leachates were determined by ICP-MS and of $\mathrm{F}$ by ESI. Values for As were higher than $1 \mathrm{mg} \mathrm{L}^{-1}$ classifying the waste as hazardous material (Class I) whereas values for $\mathrm{F}$ (> $1.5 \mathrm{mg} \mathrm{L}^{-1}$ ) were non-hazardous but indicated non-inert material (Class IIA).
\end{abstract}

Keywords: wastes; treated wood; preservatives.

\section{INTRODUÇÃO}

A madeira é empregada para diversas funções, por apresentar inúmeras vantagens e opções de utilização. Um dos seus principais usos encontra-se na construção civil, devido ao baixo consumo de energia durante o seu processamento ao ser comparada a outros materiais, como aço, cimento e alumínio. ${ }^{1}$ A origem biológica deste material, constituído essencialmente por uma matriz relativamente hidrofóbica e de fibras hidrofílicas, o diferencia de outros materiais industriais. Porém, a grande desvantagem associada a este fato é a propensão à deterioração. ${ }^{2,3}$ No ambiente terrestre, os fungos, as bactérias e os insetos são os principais agentes biológicos que destroem a madeira. Para atenuar ou até mesmo impedir a biodeterioração utilizam-se principalmente preservantes, que são substâncias químicas tóxicas aos agentes biodeterioradores. Os hidrossolúveis são os mais utilizados para esse fim e são compostos por uma associação de vários sais solúveis em água, em que as substâncias químicas presentes possuem ação inseticida e fungicida. ${ }^{4}$ Entre esses preservantes destaca-se o arseniato de cobre cromatado (CCA) utilizado em diversos países gerando, consequentemente, um grande volume de madeira tratada no mundo inteiro. ${ }^{5} \mathrm{O}$ processo de impregnação utilizando este preservante normalmente é feito empregando-se uma autoclave (processo de vácuo-pressão). Após a impregnação, a madeira é posta para secar por um período de 2 semanas, para que as reações de fixação entre o CCA e os componentes da madeira se completem. Essas reações normalmente ocorrem apenas na parte mais externa da madeira, ou seja, no alburno.

O processo de tratamento curativo (ou retratamento) é utilizado como alternativa para intervir no processo de biodeterioração em estruturas de madeira tratada com CCA e já instaladas (em serviço). Para tanto, diversos produtos são utilizados, cabendo destacar os preservantes contendo boro e flúor $(\mathrm{B} / \mathrm{F})$. A introdução deste tipo de preservante na madeira é baseada no princípio da difusão, onde os fungicidas são liberados diretamente na zona crítica, ou seja, na região em deterioração. ${ }^{1,6}$ Devido ao princípio de difusão, a impregnação da

*e-mail: suzana.ferrarini@gmail.com madeira empregando este tipo de produto ocorre externa e internamente, ou seja, no alburno e no cerne. No Brasil, este tipo de preservante tem comercialização recente, sendo aplicado por algumas companhias de distribuição de energia elétrica em postes em serviço. Um estudo pioneiro sobre o uso destes preservantes em moirões instalados em áreas de testes controladas sinalizou um provável aumento do tempo de vida útil dessas estruturas. Entretanto, a comprovação da eficácia do retratamento, em condições reais (por exemplo, postes em serviço), bem como a possível migração do F para o solo e águas adjacentes à madeira tratada, devem ser melhores avaliadas.?

Na América Latina, o Brasil aparece como o maior consumidor de madeira tratada, com aproximadamente $700.000 \mathrm{~m}^{3} /$ ano, a maior parte oriunda de florestas plantadas de eucalipto $(93,5 \%)$ e pinus $(6,5 \%)$. Deste total, a grande maioria é tratada com CCA, aplicada na produção de moirões (62\%), postes (30\%), dormentes (5\%) e para a construção civil (3\%). ${ }^{8}$

Do ponto de vista comercial, a associação de sais presentes no CCA é uma excelente alternativa para aumentar a durabilidade da madeira. Entretanto, no que diz respeito ao meio ambiente e à saúde pública seu uso é polêmico, devido ao alto índice de toxicidade apresentada. Os componentes presentes neste preservante, principalmente arsênio e cromo, são elementos com alta toxicidade e, em vários países, há restrições quanto à sua utilização. Essas restrições possuem como base a perda dos componentes do CCA ao longo do tempo por lixiviação ou volatilização, acarretando riscos de contaminação ao ser humano e ao meio ambiente. ${ }^{9}$ Além dos problemas ambientais e ocupacionais relacionados à produção e utilização da madeira tratada com CCA, um desafio ainda maior, atualmente, é a disposição final dos resíduos gerados após a vida útil, por serem considerados perigosos..$^{10,11}$

Existem diferentes interpretações no que diz respeito à classificação dos resíduos resultantes dos processos de tratamento da madeira. Em alguns países europeus, esses resíduos recebem a classificação de perigosos e, por este motivo, a sua disposição final segue legislação rigorosa. ${ }^{12}$ Em 2004, países como a Suécia e Dinamarca proibiram o uso de madeira tratada com CCA em algumas aplicações especiais como, por exemplo, usos domiciliares. Nos Estados Unidos, a Agência 
de Proteção Ambiental (EPA) declarou, em 2003, que as indústrias decidiram voluntariamente não mais empregar madeira tratada com CCA para objetos de uso residencial, devido ao alto risco de contato para os seres humanos, não havendo restrições quanto ao emprego do produto para fins rurais e industriais. ${ }^{9}$ No entanto, em outros países, como o Brasil, ainda não despertaram atenção necessária e são tratados da mesma forma que os demais resíduos sólidos, conforme a norma brasileira. ${ }^{13}$ Segundo esta norma, apenas os efluentes líquidos e resíduos originados do processo de preservação da madeira provenientes de plantas que recebem preservantes inorgânicos, contendo As ou $\mathrm{Cr}$, são classificados como resíduos perigosos. Por outro lado, não há registros na literatura em relação às implicações da possível presença de boro e flúor nos resíduos de madeira tratada com preservantes à base desses compostos. Enquanto o $\mathrm{B}$ é um micronutriente importante para o desenvolvimento das plantas, o $\mathrm{F}$ é considerado um importante poluente ambiental e sua presença em resíduos pode torná-los perigosos. ${ }^{13}$

Apesar do uso intensivo de madeira tratada com CCA no Brasil, não existem estudos sobre a classificação dos resíduos gerados no final da vida útil dessas estruturas. Também não foram relatados os possíveis impactos da presença do F em madeiras tratadas com CCA e as possíveis implicações para seu reuso, tratamento e disposição final. Desse modo, o objetivo deste trabalho foi a classificação dos resíduos de madeira tratada, de acordo com a norma brasileira ABNT NBR 10004. Para tanto, diversas amostras de postes e moirões de madeira tratados com CCA e retratados com $\mathrm{B} / \mathrm{F}$ foram retirados de serviço e submetidas a ensaios normatizados de lixiviação (ABNT NBR 10005) ${ }^{14}$ e testes de solubilização (ABNT NBR 10006). ${ }^{15}$ Os extratos obtidos foram analisados quanto à presença de $\mathrm{As}, \mathrm{Cr}$ e F. Com o intuito de obter as concentrações inicias de As, Cr e F, as amostras de madeiras foram decompostas em forno de micro-ondas. Para quantificação dos elementos investigados, em todos os testes do trabalho (lixiviação, solubilização e decomposição), foram empregadas as técnicas de espectrometria de massa com plasma indutivamente acoplado (ICP-MS), para As e Cr, e de eletrodo íon seletivo (EIS), para F.

\section{PARTE EXPERIMENTAL}

\section{Instrumentação}

A quantificação de As e Cr foi feita empregando-se um espectrômetro de massa com plasma indutivamente acoplado, modelo Elan 6000 (Perkin-Elmer). Os parâmetros instrumentais estão apresentados na Tabela 1. A otimização do instrumento foi realizada através do ajuste da vazão do gás nebulizador e alinhamento do espectrômetro de massa em relação à tocha (ajuste $\mathrm{x}-\mathrm{y}$ ) em função de se obter a máxima produção de íons $\mathrm{M}^{+}$e mínima de $\mathrm{M}^{++}, \mathrm{MO}^{+}$e sinal de fundo $m / z=220$, utilizando a nebulização convencional.

Tabela 1. Parâmetros instrumentais do ICP-MS

\begin{tabular}{lc}
\hline Parâmetros instrumentais & ICP-MS \\
\hline Vazão do gás principal & $15 \mathrm{~L} \mathrm{~min}^{-1}$ \\
Vazão do gás intermediário & $1,2 \mathrm{~L} \mathrm{~min}^{-1}$ \\
Vazão do gás de nebulização & $0,90 \mathrm{~L} \mathrm{~min}^{-1}$ \\
Potência do gerador RF & $1100 \mathrm{~W}$ \\
Varreduras por leitura & 3 \\
Leituras por replicata & 1 \\
Replicatas & 5 \\
Unidade de medida & $\mathrm{cps}$ \\
Isótopos medidos & ${ }^{75} \mathrm{As} \mathrm{e}{ }^{53} \mathrm{Cr}$ \\
\hline
\end{tabular}

Para evitar a interferência espectral do poliatômico ${ }^{40} \mathrm{Ar}^{35} \mathrm{Cl}^{+}$na determinação de ${ }^{75} \mathrm{As}$ empregou-se a equação elementar $-3,127 *$ (Se 77 - $(0,874 *$ Se 82$)$.

Utilizou-se argônio comercial com pureza de 99,996\% (White Martins) para a geração do plasma, sendo também utilizado como gás de nebulização e auxiliar.

Para a determinação da presença de F (na forma de fluoreto solúvel) empregou-se a técnica de eletrodo íon seletivo (EIS). Para tanto, utilizou-se pH-metro modificado modelo DM 20 (Digimed) equipado com eletrodo combinado de fluoreto, modelo 9609BNWP (Orion).

A agitação das amostras contendo solução extratora foi realizada empregando-se agitador de Wagner, modelo MA 160 (Marconi).

A redução de tamanho das amostras de postes de madeira para os testes de decomposição foi feita, primeiramente, com o auxílio de um formão e um martelo. Posteriormente, fez-se a moagem das referidas amostras empregando-se dois diferentes moinhos, o moinho modelo A11 IKA (Basic) e o moinho TE 600 (Tecnal).

A decomposição das amostras foi realizada em forno micro-ondas, modelo Multiwave (Anton Paar) equipado com tubos de tetrafluormetoxi (TFM).

\section{Materiais e reagentes}

Todos os reagentes empregados eram de grau analítico. Todos os frascos e vidrarias utilizados foram previamente lavados com detergente neutro Extran ${ }^{\circledR}$ a $5 \%$ e, em seguida, descontaminados com solução de $\mathrm{HNO}_{3} 10 \%$ v/v por $120 \mathrm{~h}$ ou em solução de $\mathrm{HNO}_{3}$ $50 \%$ v/v por, no mínimo, 24 h. Após a descontaminação, os frascos foram lavados e enxaguados várias vezes com água deionizada. Água deionizada purificada em sistema Milli- $\mathrm{Q}^{\circledR}$, com resistividade de 18 $\mathrm{m} \Omega \mathrm{cm}^{-1}$, foi utilizada no preparo das amostras e soluções.

As soluções de calibração dos elementos As e Cr para as leituras no ICP-MS foram preparadas a partir de diluições adequadas de soluções estoque monoelementares de $1.000 \mathrm{mg} \mathrm{L}^{-1}$ (Titrisol, Merck) e, a partir dessas soluções, foram preparadas soluções de calibração multielementares. Solução padrão do elemento ródio foi empregada como padrão interno em uma concentração de $5 \mu \mathrm{g} \mathrm{L} \mathrm{L}^{-1}$. Essa solução foi adicionada nas amostras, nos brancos e nas soluções de calibração.

Para a quantificação de $\mathrm{F}$, a curva de calibração externa foi preparada com padrões $\left(0,1 \mathrm{a} 50 \mathrm{mg} \mathrm{L}^{-1}\right.$ de fluoreto), por diluição da solução estoque $1.000 \mathrm{mg} \mathrm{L}^{-1}$ (AccuStandard ${ }^{\circledR}$ ). Uma solução TISAB (acetato de sódio $0,75 \mathrm{~mol} \mathrm{~L}^{-1}$, ácido acético $0,25 \mathrm{~mol} \mathrm{~L}^{-1}$, citrato de sódio $0,001 \mathrm{~mol} \mathrm{~L}^{-1}$ e cloreto de sódio $1,0 \mathrm{~mol} \mathrm{~L}^{-1}$ ) foi utilizada como tampão.

\section{Quantificação do preservante na madeira tratada}

A quantificação da porção inicial de As e Cr foi feita por decomposição em forno micro-ondas. A exatidão da metodologia foi avaliada pela análise do material de referência certificado de madeira SRM 1575a (Trace Elements in Pine Needles) do NIST (National Institute of Standards and Technology), procedente da Carolina do Norte. Este material foi escolhido por apresentar maior similaridade com a matriz madeira.

Foram utilizadas amostras de madeira tratada de diferentes características: postes novos submetidos à impregnação em laboratório, postes novos submetidos ao tratamento preservante na indústria e postes retirados de serviço. Para a decomposição, as amostras foram moídas com $\mathrm{CO}_{2(\mathrm{~s})}$ (gelo seco), para evitar perdas por aquecimento/ volatilização, e peneiradas (tamanho de partícula $<425 \mu \mathrm{m}$ ). As amostras foram pesadas (aproximadamente $0,10 \mathrm{~g}$ ) em balança analítica, transferidas para os frascos do micro-ondas e, após, foi adicionada 
uma mistura de $5 \mathrm{~mL}$ de $\mathrm{HNO}_{3}+2 \mathrm{~mL}$ de $\mathrm{H}_{2} \mathrm{O}_{2}$. As amostras foram submetidas ao programa de aquecimento apresentado na Tabela 2. Após resfriamento, as soluções foram transferidas quantitativamente para frascos de polipropileno e o volume ajustado a $50 \mathrm{~mL}$ com água deionizada. As amostras foram preparadas em triplicata.

Tabela 2. Programa de decomposição em forno de micro-ondas

\begin{tabular}{ccc}
\hline Etapa & Tempo & Temperatura atingida $\left({ }^{\circ} \mathrm{C}\right)$ \\
\hline 1 & 2 min e $30 \mathrm{~s}$ & 90 \\
2 & 5 min & 130 \\
3 & 3 min e $30 \mathrm{~s}$ & 200 \\
4 & 15 min & 200 \\
Resfriamento & - & - \\
\hline
\end{tabular}

\section{Ensaio de lixiviação - ABNT NBR 10005 ${ }^{14}$}

Para a realização do teste de classificação do resíduo contendo CCA (As e Cr) foram selecionadas 6 amostras de postes retirados de serviço, utilizando-se apenas a região do alburno, e outras 6 englobando toda a região (cerne e alburno). A redução de tamanho das amostras foi feita com o auxílio de formão e martelo. Posteriormente, foram peneiradas (peneira com abertura de malha $\leq$ 9,5 mm de diâmetro). A solução extratora foi definida com base na medida do $\mathrm{pH}$ da solução. Esta foi preparada pela adição de ácido acético glacial e hidróxido de sódio $1,0 \mathrm{~mol} \mathrm{~L}^{-1} \mathrm{e}$, posteriormente, o volume foi acertado com água deionizada. A massa de amostra empregada foi 20 vezes menor que o volume de solução extratora. Amostra e solução extratora foram transferidas para frascos de borosilicato e, então, colocadas em agitação em agitador tipo Wagner a $30 \mathrm{rpm}$, por um período de $18 \pm 2 \mathrm{~h}$. Após este procedimento, as amostras foram submetidas à filtração a vácuo através de membranas de fibra de vidro, isentas de resinas, com porosidade de 0,6 a 0,8 $\mu \mathrm{m}$. Para conservação das amostras, quando não eram analisadas imediatamente por ICP-MS, após determinação do $\mathrm{pH}$ no extrato lixiviado resultante, era feito um ajuste de $\mathrm{pH}$ (aproximadamente 2), com $\mathrm{HNO}_{3}$ concentrado.

Para a realização do teste de classificação do resíduo contendo $\mathrm{CCA}+\mathrm{B} / \mathrm{F}(\mathrm{F})$, foram selecionadas 9 amostras utilizando as regiões cerne e alburno: 2 moirões coletados na área de teste controlada em Canoas/RS, ambos contendo B e F, e 7 postes retirados de serviço que foram submetidos ao retratamento. A preparação das amostras e o ensaio de lixiviação foram executados da mesma maneira que o teste de classificação para os resíduos contendo CCA (As e Cr), porém, imediatamente após a filtração, o fluoreto solúvel era medido utilizando-se $10 \mathrm{~mL}$ da solução obtida e $10 \mathrm{~mL}$ de solução tampão TISAB e a leitura feita por EIS.

\section{Ensaios de solubilização - ABNT NBR 10006 ${ }^{15}$}

Primeiramente, as amostras de madeira (tamanho $\leq 9,5 \mathrm{~mm}$ de diâmetro) que receberam tratamento curativo foram submetidas à secagem em estufa com circulação forçada de ar e exaustão em uma temperatura de até $42^{\circ} \mathrm{C}$. Posteriormente, uma massa representativa (base seca) foi transferida para frasco de borossilicato, onde o volume foi completado com água deionizada. Após, a amostra foi agitada em agitador tipo Wagner por um período de $5 \mathrm{~min}$, em velocidade baixa e, em seguida, houve a vedação do frasco com filme de PVC. A amostra foi então deixada em repouso por um período de 7 dias, em temperatura de até $25^{\circ} \mathrm{C}$. Após este período, a solução resultante foi filtrada (filtro de membrana com porosidade $0,45 \mu \mathrm{m}$, Millipore) e a concentração de fluoreto medida por EIS.

\section{RESULTADOS E DISCUSSÃO}

\section{Quantificação de As e Cr na madeira tratada}

Os resultados referentes à quantificação de As e Cr estão apresentados na Tabela 3. Pela análise da tabela pode-se observar que as concentrações obtidas para As e Cr dependem do tipo de amostra analisada. Em amostras de postes novos, as concentrações destes elementos são bem maiores. Este fato já era esperado, uma vez que neste tipo de amostra os referidos elementos encontram-se mais fixados na madeira e ainda não foram submetidos a nenhuma condição de intempérie, que provocaria a lixiviação dos mesmos. Nessas amostras, a concentração média encontrada para As e Cr foi de 2923

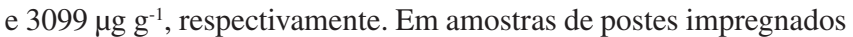
em laboratório, a concentração dos analitos foi menor em comparação com a de postes impregnados industrialmente (postes novos). Essa redução é coerente, uma vez que a impregnação industrial utiliza uma solução preservante contendo em média 3,5\% (m/m) dos princípios ativos e a impregnação em laboratório utilizou uma concentração de $1 \%(\mathrm{~m} / \mathrm{m})$. Para essas amostras a concentração média obtida para As foi de $797 \mu \mathrm{g} \mathrm{g}^{-1}$ e para $\mathrm{Cr} 1046 \mu \mathrm{g} \mathrm{g}^{-1}$. Cabe destacar nesse tipo de amostra, as diferentes faixas de concentrações obtidas entre as diferentes espécies de madeira empregadas (C. citriodora, E. saligna e E. grandis) possivelmente estão relacionadas às diferentes densidades e umidades dessas espécies, que fazem com que a impregnação seja mais ou menos eficiente. ${ }^{16}$ As concentrações obtidas na amostra de poste retirado de serviço, como já esperado, foram bem menores dos que as obtidas nas outras amostras. Esses resultados indicam uma perda dos analitos para o ambiente, devido à volatilização e lixiviação dos mesmos.

Tabela 3. Análise de amostras de madeira tratada com CCA por ICP-MS. Decomposição em forno micro-ondas; mistura ácida: $5 \mathrm{~mL}$ de $\mathrm{HNO}_{3}$ conc. $+2 \mathrm{~mL}$ de $\mathrm{H}_{2} \mathrm{O}_{2}$. Tamanho de partícula $\leq 425 \mu \mathrm{m}$

\begin{tabular}{ccc}
\hline Amostra/tempo de serviço/espécie & Elemento & $\begin{array}{c}\text { Conc. } \\
\left(\mu \mathrm{g} \mathrm{g}^{-1}\right)\end{array}$ \\
\hline Poste retirado de serviço/ & $\mathrm{As}$ & $479(9)$ \\
15 anos/E. citriodora & $\mathrm{Cr}$ & $1100(2)$ \\
\hline Poste novo 1/Preservado & $\mathrm{As}$ & $3004(143)$ \\
em 08/2008/E. saligna & $\mathrm{Cr}$ & $2947(149)$ \\
Poste novo 2/Preservado & $\mathrm{As}$ & $4229(32)$ \\
em 08/2008/E. saligna & $\mathrm{Cr}$ & $4533(49)$ \\
Poste novo 3/Preservado & $\mathrm{As}$ & $1538(70)$ \\
em 08/2008/E. saligna & $\mathrm{Cr}$ & $1819(78)$ \\
\hline Poste impregnado/Impregnado & $\mathrm{As}$ & $670(6)$ \\
em 07/2009/C. citriodora & $\mathrm{Cr}$ & $869(16)$ \\
\hline Poste impregnado/Impregnado & $\mathrm{As}$ & $535(16)$ \\
em 07/2009/E. saligna & $\mathrm{Cr}$ & $707(29)$ \\
\hline Poste impregnado/Impregnado & $\mathrm{As}$ & $1188(60)$ \\
em 07/2009/E. grandis & $\mathrm{Cr}$ & $1564(74)$ \\
\hline
\end{tabular}

Outro ponto fundamental que merece destaque é a importância do estudo de classificação e descontaminação ser realizado empregando amostras de postes retirados de serviço, porque são efetivamente as que melhor representam os resíduos de madeira. Mesmo com todos os problemas relacionados à falta de homogeneidade deste tipo de amostras, ${ }^{17}$ as mesmas representam a real situação em que se encontram os postes atualmente. 


\section{Ensaios de lixiviação para classificação dos resíduos (As e Cr)}

A Tabela 4 apresenta as concentrações obtidas para os elementos As e $\mathrm{Cr}$ no ensaio de lixiviação para os postes dos quais se utilizou somente a parte do alburno.

Tabela 4. Concentrações obtidas para As e Cr no extrato lixiviado (somente alburno) por ICP-MS ao se aplicar o ensaio de lixiviação segundo ABNT NBR 10005

\begin{tabular}{ccc}
\hline Amostra/Espécie & $\begin{array}{c}\text { Concentração Cr } \\
\left(\mathrm{mg} \mathrm{L}^{-1}\right)\end{array}$ & $\begin{array}{c}\text { Concentração As } \\
\left(\mathrm{mg} \mathrm{L}^{-1}\right)\end{array}$ \\
\hline Poste A/E. citriodora & 1,5 & 5,6 \\
Poste B/E. grandis & 1,9 & 2,7 \\
Poste C/E. grandis & 6,1 & 4,0 \\
Poste D/E. grandis & 1,0 & 9,2 \\
Poste E/E. grandis & 1,2 & 7,3 \\
Poste F/E. grandis & 0,6 & 7,5 \\
\hline
\end{tabular}

Limite máximo no extrato após ensaio de lixiviação ABNT NBR 10004 5,0 1,0

Observa-se na Tabela 4 que a concentração obtida para As variou de 2,7 a 9,2 mg L L $^{-1}$. Nos 6 postes analisados a concentração deste elemento ultrapassou $1 \mathrm{mg} \mathrm{L}^{-1}$, isto significa que este material pode ser considerado como resíduo perigoso se a regulamentação da ABNT NBR 10004 for aplicada para resíduos de madeiras tratadas com CCA. Segundo propriedades de toxicidade, esse tipo de resíduo pode ser considerado como resíduo perigoso - classe I. Com relação ao elemento $\mathrm{Cr}$, observa-se que apenas em um dos postes o valor de concentração encontrado ultrapassou o limite regulamentado pela ABNT NBR 10004, de 5,0 mg L-1.

Ainda no sentido de comprovar que os testes de lixiviação classificam esse tipo de resíduo como perigoso, foi realizado mais um ensaio de lixiviação, porém agora empregando uma mistura de cerne e alburno, ao invés de apenas alburno. A Tabela 5 mostra uma comparação entre os resultados obtidos utilizando apenas alburno e a mistura cerne e alburno.

Tabela 5. Comparação dos resultados obtidos para As e Cr no extrato lixiviado por ICP-MS, utilizando somente alburno e a mistura de cerne e alburno

\begin{tabular}{ccc}
\hline Amostra/Espécie & $\begin{array}{c}\text { Concentração } \\
\mathrm{Cr} \\
\left(\mathrm{mg} \mathrm{L}^{-1}\right)\end{array}$ & $\begin{array}{c}\text { Concentração } \\
\text { As } \\
\left(\mathrm{mg} \mathrm{L}^{-1}\right)\end{array}$ \\
\hline Poste F (A) $/ E$. grandis & 0,6 & 7,5 \\
Poste F $(\mathrm{C}+\mathrm{A})^{\mathrm{b}} / E$. grandis & 0,4 & 3,5 \\
Poste $\mathrm{G}(\mathrm{C}+\mathrm{A}) / E$. grandis & 0,2 & 1,1 \\
Poste H $(\mathrm{C}+\mathrm{A}) / E$. grandis & 0,2 & 2,3 \\
Poste I $(\mathrm{C}+\mathrm{A}) / E$. grandis & 0,1 & 1,2 \\
Poste J $(\mathrm{C}+\mathrm{A}) / E$. grandis & 0,5 & 1,8 \\
\hline Poste L $(\mathrm{C}+\mathrm{A}) /$ Espécie desconhecida & 0,2 & 3,4 \\
\hline Poste M $(\mathrm{C}+\mathrm{A}) /$ Espécie desconhecida & 1,5 & 6,2 \\
\hline Limite máximo no extrato após ensaio de lixiviação ABNT NBR 10004 \\
\hline
\end{tabular}

${ }^{\mathrm{a}}$ somente alburno; ${ }^{\mathrm{b}}$ mistura cerne e alburno

Observa-se que mesmo utilizando a mistura cerne e alburno, o resíduo de madeira preservada com CCA (segundo dados obtidos para As) continua sendo classificado como perigoso, pelas características de toxicidade. A única diferença observada quando se emprega a mistura cerne e alburno é a diminuição na concentração dos elementos, com exceção da concentração de $\mathrm{Cr}$ no poste $\mathrm{M}$. Ao se comparar os dados obtidos para a mesma amostra (amostra F), quando se emprega a mistura, tem-se uma diminuição nas concentrações dos dois elementos em aproximadamente $50 \%$. Este fato já era esperado, pois ao se utilizar a mistura tem-se uma espécie de "diluição" da amostra. No cerne espera-se uma quantidade bem menor dos elementos, uma vez que o tratamento preservante nesta região é bem menos eficiente.

Os resultados obtidos por Silva ${ }^{10}$ em duas espécies diferentes (Pinus e Eucalipto) de madeira recém-submetida ao tratamento com CCA, também classificam esse tipo de resíduo como perigoso. Neste trabalho, os valores de concentração para As variaram de 16,5 a 32,0 $\mathrm{mg} \mathrm{L}^{-1}$ e para $\mathrm{Cr}$, de 7,0 a 14,7 $\mathrm{mg} \mathrm{L}^{-1}$. Os resultados obtidos por esse autor foram superiores aos encontrados no presente estudo, uma vez que em um poste recém-tratado as quantidades de Cr e As são significativamente maiores, pois esses postes ainda não foram submetidos a nenhuma condição de uso ou mesmo sujeitos às intempéries. Cabe ainda destacar que, em um poste recém-submetido ao tratamento preservante, as reações de fixação do preservante com a madeira ainda não são completas facilitando, desta forma, a sua remoção e, consequentemente, a concentração dos elementos presentes no lixiviado será bem maior.

O teste de lixiviação empregado também pode ser interpretado pela norma americana da EPA - Agência de Proteção Ambiental. Segundo a EPA, esse teste é conhecido como TCLP - procedimento de lixiviação para características de toxicidade (Método 1311). ${ }^{18}$ Segundo a literatura, ${ }^{17}$ se a concentração do elemento As presente no extrato lixiviado for superior a $5 \mathrm{mg} \mathrm{L}^{-1}$, o resíduo é então classificado como sendo perigoso. Esse mesmo autor aplicou este teste em 4 amostras de madeira tratada com CCA obtendo valores (para As) que variaram de 4,3 a 9,2 $\mathrm{mg} \mathrm{L}^{-1}$. Das 4 amostras testadas, o valor limite para As foi ultrapassado em 3, caracterizando desta forma o resíduo como perigoso. Townsend et al. ${ }^{19}$ aplicaram o mesmo teste neste tipo de resíduo e, em 11 das 13 amostras testadas, o limite para As foi ultrapassado.

Para os resíduos de madeira analisados no presente trabalho, os resultados determinados para As, no extrato lixiviado, os classificam como perigosos, de acordo com a norma brasileira. Por outro lado, conforme a norma americana, apenas os resíduos de 2 amostras, Poste F (A) e Poste M (C+A), seriam classificados como perigosos.

\section{Ensaios de lixiviação para classificação dos resíduos contendo $\mathbf{F}$}

O ensaio de lixiviação foi aplicado em amostras de postes de madeira preservada com CCA e que, posteriormente, receberam tratamento curativo com preservante contendo F. A ABNT NBR 10004 estabelece que a concentração do referido elemento, expressa como fluoreto ( $\left.\mathrm{F}^{-}\right)$, não deve ultrapassar $150 \mathrm{mg} \mathrm{L}^{-1}$ no extrato lixiviado. A realização deste teste de classificação em função deste elemento é de grande importância uma vez que, futuramente, haverá uma grande quantidade de postes retirados com a presença do mesmo. A Tabela 6 apresenta os resultados da concentração de $\mathrm{F}^{-}$, em $\mathrm{mg} \mathrm{L}^{-1}$, presente nos extratos lixiviados das amostras.

Como pode ser observado nesta tabela, as concentrações obtidas para o elemento em estudo (em todas as amostras) não ultrapassaram os limites estabelecidos pela ABNT NBR 10004, de $150 \mathrm{mg} \mathrm{L}^{-1}$. Desta forma, esse tipo de resíduo não é considerado perigoso e sim, resíduo não perigoso classe II.

As variações nas concentrações observadas entre as amostras podem estar associadas ao tempo que estes postes ficaram em serviço e à não homogeneidade de sua distribuição na madeira. Os postes que apresentaram concentrações menores de fluoreto permaneceram um 
Tabela 6. Concentrações obtidas para fluoreto $\left(\mathrm{F}^{-}\right)$no extrato lixiviado por EIS (cerne e alburno) ao se aplicar ensaio de lixiviação segundo ABNT NBR 10005 (2004)

\begin{tabular}{cc}
\hline Amostra/Espécie & Concentração F $\left(\mathrm{mg} \mathrm{L}^{-1}\right)$ \\
\hline Poste 1/E. grandis & 45 \\
Poste 2/E. grandis & 2 \\
Poste 3/E. grandis & 14 \\
Poste 4/E. grandis & 4 \\
Poste 5/E. grandis & 61 \\
Poste 6/Espécie desconhecida & 15 \\
Poste 7/Espécie desconhecida & 2 \\
Moirão 3/E. citriodora & 14 \\
Moirão 10/E. citriodora & 7 \\
\hline Limite máximo no extrato após ensaio de lixiviação ABNT NBR 10004 \\
\hline
\end{tabular}

período maior em serviço e, consequentemente, podem ter ocorrido maiores perdas para o solo.

\section{Teste de solubilização para classificação dos resíduos contendo F}

Como já discutido, os resíduos de postes de madeira que receberam tratamento curativo não foram considerados perigosos, de acordo com a norma de classificação. Porém, para ser corretamente descartado e não necessitar de envio para aterro sanitário, o resíduo não perigoso classe II necessita de mais um teste que o caracterize como resíduo classe II B - inerte. Este teste é conhecido como teste de solubilização. Na Tabela 7, são mostrados os resultados obtidos com a aplicação do mesmo.

Tabela 7. Concentrações obtidas para fluoreto ( $\left.\mathrm{F}^{-}\right)$no extrato solubilizado por EIS (cerne e alburno) ao se aplicar teste de solubilização de resíduos sólidos segundo ABNT NBR 10006 (2004)

\begin{tabular}{cc}
\hline Amostra/Espécie & Concentração $\mathrm{F}^{-}\left(\mathrm{mg} \mathrm{L}^{-1}\right)$ \\
\hline Poste $5(1) /$ E. grandis & 24 \\
Poste 5 (2)/E. grandis & 27 \\
\hline
\end{tabular}

Limite máximo no extrato após ensaio de solubilização ABNT NBR 10004

1,5

De acordo com a Tabela 7, observa-se que a concentração obtida para fluoreto foi aproximadamente 17 vezes maior que o limite estabelecido pela ABNT NBR 10006. Desta forma, segundo resultados obtidos com o referido teste, os resíduos de postes de madeira que receberam tratamento curativo são considerados resíduos classe II A - não inerte - por apresentarem concentrações de fluoreto superiores aos padrões de potabilidade de água. Com esta classificação, esse tipo de resíduo não pode ser descartado de qualquer forma ou em qualquer lugar; precisa, necessariamente, ser enviado para um aterro.

\section{CONCLUSÃO}

Os resultados obtidos com a aplicação dos ensaios de lixiviação em resíduos de madeira preservada com CCA mostram que estes resíduos devem ser classificados como classe I - perigosos, de acordo com a regulamentação da ABNT NBR 10004, onde o limite máximo para As (1 $\left.\mathrm{mg} \mathrm{L}^{-1}\right)$ foi ultrapassado em todas as amostras analisadas de postes retirados de serviço. Também merecem atenção os resíduos dos postes retratados com $\mathrm{F}$, que ultrapassaram o limite de $1,5 \mathrm{mg}$ $\mathrm{L}^{-1}$ e, consequentemente, receberam a classificação de não inertes. Com base nas classificações obtidas para os resíduos analisados, os mesmos não podem ser descartados de qualquer forma ou em qualquer lugar. Eles deveriam, necessariamente, ser enviados para um aterro especial ou passarem por um processo de remoção dos constituintes considerados perigosos. A remoção dos elementos pode ser vantajosa, uma vez que, neste processo, se obtém um resíduo sólido descontaminado que pode ser reutilizado para fins industriais, como fonte de energia, ou descartado em aterro comum, e um efluente líquido rico em $\mathrm{Cu}, \mathrm{Cr}$ e As, os quais podem ser recuperados por processo de precipitação química e utilizados para fins industriais nobres, uma vez que possuem elevada pureza, ${ }^{20}$ Devido ao crescente aumento desses resíduos no futuro, torna-se essencial o desenvolvimento de metodologias adequadas para uma correta disposição final dos mesmos.

\section{AGRADECIMENTOS}

\section{À AES Sul, ao CNPq, à FAPERGS e CAPES.}

\section{REFERÊNCIAS}

1. Vidor, F. L. R.; Pires, M. J. R.; Dedavid, B. A.; Montani, P. D. B.; Gabiatti, A.; IEEE Transactions on Power Delivery 2010, 25, 479.

2. Clausen, C. A.; Int. Biodeterirations \& Biodegradation 1996, 37, 101.

3. Kin, Y. S.; Singh, A. P.; Iana J. 2000, 21, 135.

4. Jang, Y. C.; Townsend, T. G.; Ward, M.; Bitton, G.; Environmental Contamination and Toxicology 2002, 69, 808.

5. http://www.twbrazil.com.br/upload/arquivos/179f6e8817.pdf, acessada em Agosto 2012.

6. Lepage, E. S.; Manual de Preservação de Madeiras, IPT - Divisão de Madeiras: São Paulo, 1986, vols. I e II.

7. Vidor, F. L. R.; Tese de Doutorado, Pontifícia Universidade Católica do Rio Grande do Sul, Brasil, 2011.

8. Silva, J. C.; Revista da Madeira 2006, 16, 100.

9. Moreschi, J. C.; Rocha, M. P.; Borges, C. C.; Carvalho, R. C.; Revista da Madeira 2002, 68, 12.

10. Silva, G. A. da; Dissertação de Mestrado, Instituto de Pesquisas Tecnológicas do Estado de São Paulo, Brasil, 2008.

11. Ferrarini, S. F.; Santos, H. S. dos; Miranda, L. G.; Pires, M. J. R.; Maia, S. M.; Azevedo, C. M. N.; Resumos do Congreso Internacional de Distribución Eléctrica, Buenos Aires, Argentina, 2010.

12. Helsen, L.; van den Bulk, E.; Environ. Pollut. 2005, 134, 301.

13. ABNT - Associação Brasileira de Normas Técnicas; Resíduos Sólidos Classificação, NBR 10004, Rio de Janeiro, 2004.

14. ABNT - Associação Brasileira de Normas Técnicas; Procedimento para Obtenção de Extrato Lixiviado de Resíduos Sólidos, Rio de Janeiro, 2004.

15. ABNT - Associação Brasileira de Normas Técnicas; Procedimento para Obtenção de Extrato Solubilizado de Resíduos Sólidos, Rio de Janeiro, 2004.

16. Santos, H. S. dos; Dissertação de Mestrado, Pontifícia Universidade Católica do Rio Grande do Sul, Brasil, 2010.

17. Janin, A.; Blais, J. F.; Drogui, P.; J. Hazard. Mater. 2009, 169, 136.

18. EPA - Environmental Protection Agency United States; Toxicity Characteristic Leaching Procedure, EPA SW - 846 - Method 1311, 1992.

19. Townsend, T.; Tolaymat, T.; Solo-Gabriele, H. M.; Dubey; B.; Stook, K.; Wadanambi, L.; J. Hazard. Mater. 2004, 114, 75.

20. Janin, A.; Blais, J. F.; Mercier, G.; Drogui, P.; J. Hazard. Mater. 2009 , 169,1099 